Special Issue: Direct Reprogramming

Original Article

\title{
Rapid cell-fate conversion of mouse fibroblasts into hepatocyte-like cells
}

\author{
Shizuka Miura" and Atsushi Suzuki ${ }^{1,2, *)}$ \\ ${ }^{1)}$ Division of Organogenesis and Regeneration, Medical Institute of Bioregulation, Kyushu University, Fukuoka, Japan \\ ${ }^{2)}$ Core Research for Evolutional Science and Technology (CREST), Japan Science and Technology Agency, Saitama, \\ Japan
}

Recent progress in studies on direct cell-fate conversion of differentiated somatic cells into other cell types, which is known as "direct reprogramming", is expected to lead to innovations in health care. In our previous study, we found that three specific combinations of two transcription factors, comprising Hnf4a plus Foxa1, Foxa2, or Foxa3, were able to induce conversion of mouse fibroblasts into functional hepatocyte-like cells. These induced hepatocyte-like (iHep) cells will be useful for developing regenerative therapies for liver diseases and examining the pharmacological effects of drugs. However, to evaluate the potential utility of iHep cells, the phenomena involved in the direct conversion of fibroblasts into iHep cells should be examined in detail. Thus, in this study, we sequentially analyzed the early stage of fibroblast conversion into iHep cells after infection with retroviruses expressing $\mathrm{Hnf} 4 \mathrm{a}$ and Foxa3. Our data demonstrated that the conversion into iHep cells began within 2 days after introduction of the transgenes into fibroblasts, and the number of iHep cells increased gradually as the culture progressed. The rapid cell-fate conversion of fibroblasts into iHep cells and stable expansion of iHep cells are two pieces of evidence suggesting the utility of iHep cells for cell transplantation therapy, bioartificial liver development, and screening of drugs for patients with liver diseases, which require many hepatocytes within a short period of time.

Rec.9/12/2014, Acc.10/23/2014, pp211-216

\footnotetext{
${ }^{*}$ Correspondence should be addressed to:

Atsushi Suzuki, Division of Organogenesis and Regeneration, Medical Institute of Bioregulation, Kyushu University, 3-1-1 Maidashi, Higashi-ku, Fukuoka 812-8582, Japan. Phone/Fax: +81-92-642-6793, E-mail: suzukicks@bioreg.kyushu-u.ac.jp
}

Key words liver, hepatocyte, iHep cell, reprogramming, fibroblast

\section{Introduction}

Hepatocytes, which compose nearly $80 \%$ of the liver mass, play important roles in the maintenance of its vital functions, including protein synthesis, detoxification, lipid metabolism, and carbohydrate metabolism. Thus, for treatment of liver diseases by cell-based transplantation 
therapies, large numbers of hepatocytes need to be prepared and used as donor cells. In addition, for screening of drugs and studies on the mechanisms of liver diseases, hepatocytes should be functionally maintained in culture with the ability to proliferate. However, it is difficult not only to isolate many hepatocytes from patients with liver diseases, but also to maintain multiple hepatocyte properties in culture. Thus, for realization of cell-based regenerative medicine for liver disorders and research on the pharmacological effects of drugs and mechanisms of liver diseases, many studies involving the generation of functional hepatocyte-like cells from non-hepatic cells are now in progress. Recently, in addition to embryonic stem cells, induced pluripotent stem (iPS) cells have been considered as the most likely source of hepatocyte-like cells, and many studies are actively underway to evaluate the utility of stem cell-derived hepatocyte-like cells ${ }^{1-6)}$.

There is, however, another approach being investigated. Direct cell-fate conversion of one cell type into another cell type without going through the iPS cell state is known as "direct reprogramming", and is currently being extensively studied worldwide as a new technology for future innovative medicine. We previously reported that the introduction of genes encoding Hnf4a plus Foxa1, Foxa2, or Foxa3 into mouse fibroblasts enabled their direct conversion into hepatocyte-like cells, termed iHep cells ${ }^{7}$. The iHep cells possessed multiple hepatocyte-specific features and could repopulate hepatic tissues after transplantation into the livers of fumarylacetoacetate hydrolase-deficient mice, a mouse model of hereditary tyrosinemia type $\mathrm{I}^{7}$. Moreover, we recently showed that iHep cells had the properties of hepatocytes in lipid metabolism, including lipid synthesis from fatty acids, cytoplasmic accumulation of lipid droplets, lipid secretion into the culture medium, and responsiveness to lipid metabolism improvers, even after culture for a relatively long period ${ }^{8)}$. Thus, it is expected that the direct reprogramming of fibroblasts to iHep cells should be studied in greater detail, and that this new technology should be improved to provide iHep cells applicable to the fields of medical science and drug discovery research, as an alternative to hepatocytes.

For use of iHep cells in therapeutic applications, direct reprogramming of fibroblasts to iHep cells needs to occur within a short period of time, because patients with liver diseases require large numbers of iHep cells as soon as possible and the cost of the associated work should be suppressed as much as possible. However, the timing for when fibroblasts start to undergo conversion into iHep cells after induction by forced expression of defined transcription factors remains largely unknown. Thus, in this study, we sequentially analyzed the early stage of the direct conversion of mouse embryonic fibroblasts (MEFs) into iHep cells using live-cell imaging, immunofluorescence staining, and quantitative polymerase chain reaction (qPCR) analyses after infection with retroviruses expressing $\mathrm{Hnf4a}$ and Foxa3.

\section{Materials and Methods}

1)Induction of iHep cells from MEFs

We induced direct conversion of MEFs into iHep cells as described previously ${ }^{7}$. Some modifications were added to the methods as described below. In the production of recombinant retroviruses, we used PLAT-E cells for transfection of plasmid DNA ${ }^{9}$. After the retrovirus infection, MEFs were cultured in our hepato-medium containing 20 $\mathrm{ng} / \mathrm{mL}$ hepatocyte growth factor (PeproTech, Rocky Hill, NJ, USA) and $20 \mathrm{ng} / \mathrm{mL}$ epidermal growth factor (Sigma-Aldrich, St. Louis, MO, USA).

\section{2)Live-cell imaging analysis}

After MEFs were infected with the retroviruses expressing Hnf4a and Foxa3, the culture dishes were set on a Nikon BioStation IM (Nikon, Tokyo, Japan), and subjected to live-cell imaging analyses for 8 days at $37^{\circ} \mathrm{C}$ under $5 \%$ $\mathrm{CO}_{2}$. Snapshots were obtained from the movies using NIS Elements software (Nikon).

\section{3)Immunofluorescence staining and gene expression analysis}

Immunofluorescence staining and qPCR analyses were conducted as described previously ${ }^{7}$. We used a rat antithymus cell antigen (Thy)-1 antibody (BioLegend, San Diego, CA, USA) to examine Thy-1 expression.

\section{Results}

1)Live-cell imaging analysis of the conversion of fibroblasts into epithelial-like cells

In this study, we analyzed the early stage of the direct conversion of MEFs into iHep cells in detail. As shown in Figure 1, we observed the morphology of MEFs using a live-cell imaging system for 8 days after infection with the retroviruses expressing $\mathrm{Hnf} 4 \mathrm{a}$ and Foxa3. We also performed immunofluorescence staining for E-cadherin and albumin, which are markers of epithelial cells and 


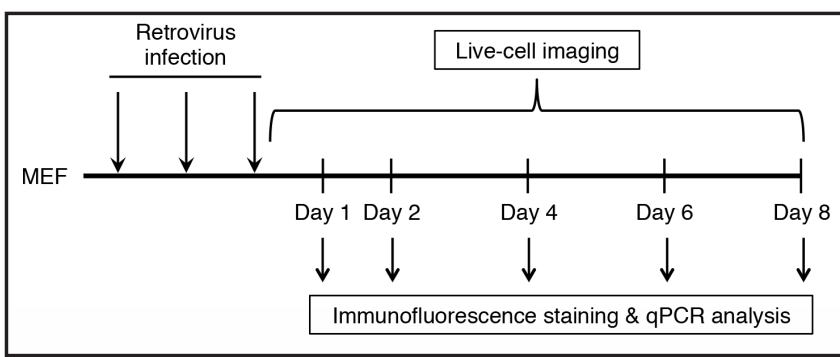

Fig.1 Schematic diagram of the experimental procedure

We conducted live-cell imaging, immunofluorescence staining, and qPCR analyses on the MEF cultures at 1, 2, 4, 6, and 8 days after infection with the retroviruses expressing Hnf4a and Foxa3.

hepatocytes, respectively, at 1, 2, 4, 6, and 8 days after the retrovirus infection. In addition, we sequentially examined the expression levels of E-cadherin and albumin and the immature hepatocyte marker $a$-fetoprotein (AFP) at 1, 2, 4, 6 , and 8 days after the retrovirus infection.

The live-cell imaging analyses revealed that normal MEFs became flattened, increased in size, and gradually became senescent, while no epithelial-like cells were observed during the analysis for 8 days (Fig. 2A). However, MEFs expressing exogenous $\mathrm{Hnf} 4 \mathrm{a}$ and Foxa3 began decreasing their size at $36 \mathrm{~h}$ after the retrovirus infection, and small, round-shaped cells appeared to increase during culture (Fig. 2B). At $72 \mathrm{~h}$ after the retrovirus infection, we observed compact cell-cell adhesion in the increased small, round-shaped cells, and they began to form small clusters of epithelial-like cells (Fig. 2B). The epithelial-like cells continued proliferation and eventually formed large clusters, and the small clusters were also bonded to each other (Fig. 2B). These data from the live-cell imaging analyses demonstrated that MEFs could initiate conversion into epithelial-like cells within 2 days after infection with the retroviruses expressing $\mathrm{Hnf} 4 \mathrm{a}$ and Foxa3, and that these cells could actively proliferate to form epithelial clusters in culture.

\section{2)Rapid induction of the expressions of markers} for epithelial cells and hepatocytes in cultures of retrovirus-infected MEFs

Next, we examined the expressions of markers for epithelial cells and hepatocytes in the early stage of the direct conversion of MEFs into epithelial-like cells. As shown in Figure 3, immunofluorescence analyses revealed that a small number of transduced MEFs initially expressed E-cadherin on their plasma membranes at 2 days after the

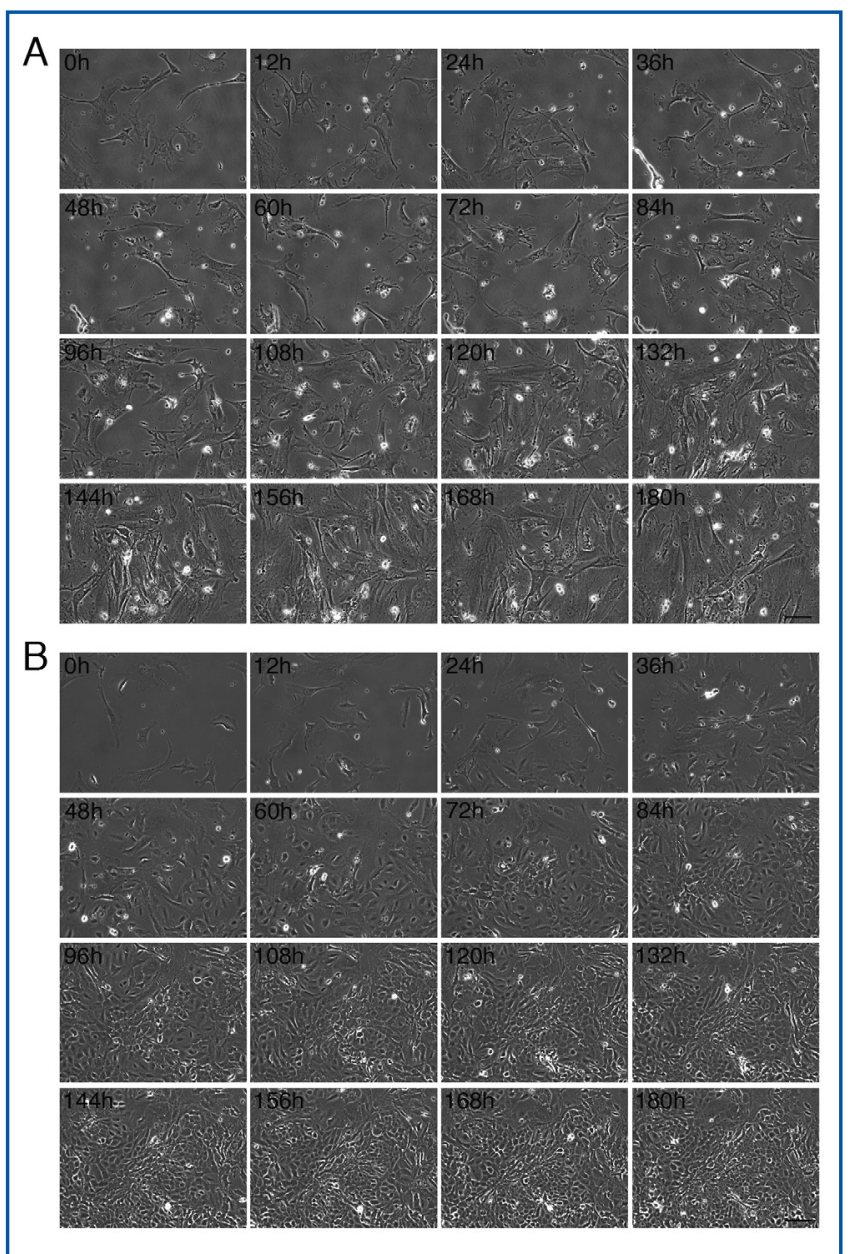

Fig.2 Live-cell imaging analyses in cultures of retrovirusinfected MEFs show rapid conversion of fibroblasts into epithelial-like cells

(A, B) Snapshots obtained from the data of live-cell imaging analyses in cultures of MEFs (A) and retrovirus-infected MEFs (B) for 8 days after infection with the retroviruses expressing $\mathrm{Hnf} 4 \mathrm{a}$ and Foxa3. Scale bars, $100 \mu \mathrm{m}$.

retrovirus infection. At the same time, albumin expression was also detected, but only faintly. At 4 days after the retrovirus infection, we could clearly detect E-cadherinpositive cells that coexpressed albumin, and the number of these cells gradually increased as the time elapsed. Interestingly, almost all E-cadherin-positive cells that appeared at 2 days after the retrovirus infection lacked the expression of the fibroblast marker Thy-1 (Fig. 4). The data obtained from GPCR analyses also showed that the expressions of E-cadherin, albumin, and AFP were detected in the MEF cultures at 2 days, 1 day, and 1 day after the retrovirus infection, respectively, and thereafter increased continuously in a time-dependent manner (Fig. 5). Taken together, direct conversion of MEFs into epithelial-like cells 


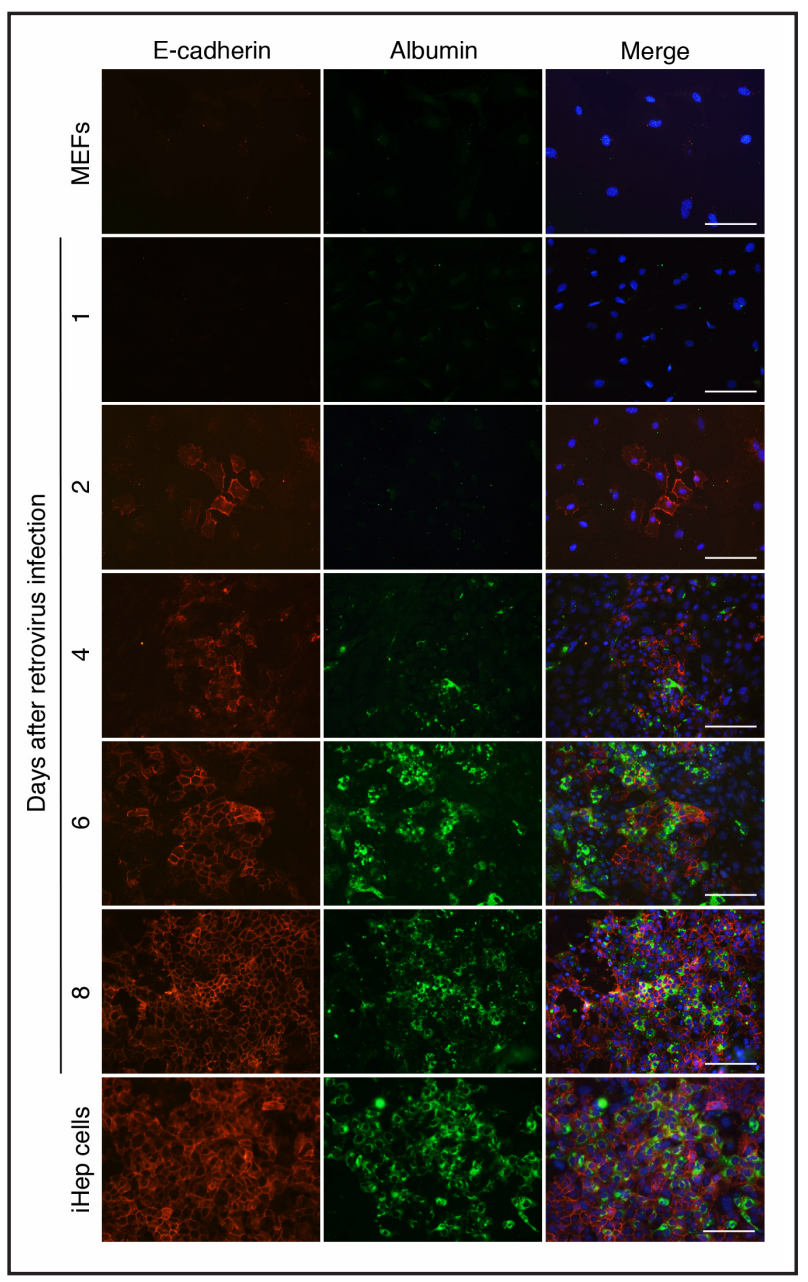

Fig.3 E-cadherin and albumin both quickly start to be expressed in cultures of retrovirus-infected MEFs

We conducted coimmunofluorescence staining for E-cadherin and albumin in MEF cultures at 1,2, 4, 6, and 8 days after infection with the retroviruses expressing Hnf4a and Foxa3. MEFs and iHep cells maintained in cultures were analyzed as negative and positive controls, respectively. DNA was stained with DAPI. Scale bars, 100 $\mu \mathrm{m}$.

with the properties of iHep cells initially occurred within 2 days after infection with the retroviruses expressing $\mathrm{Hnf} 4 \mathrm{a}$ and Foxa3.

\section{Discussion}

In our previous paper, we reported that clusters of E-cadherin-positive iHep cells were observed in MEF cultures at several weeks after infection with retroviruses expressing $\mathrm{Hnf} 4 \mathrm{a}$ and $\mathrm{Foxa}^{7}{ }^{7}$. However, the timing for when the transduced MEFs started to undergo conversion into iHep cells remained unclear. The present data obtained from live-cell imaging analyses demonstrated that the morphological changes of MEFs and the formation of

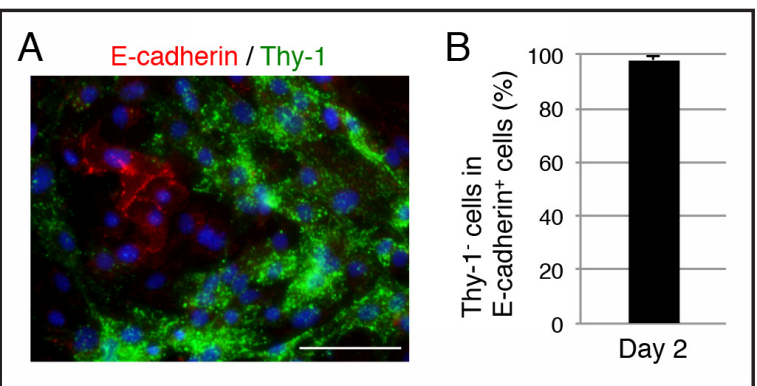

Fig.4 MEFs converting into E-cadherin-positive cells simultaneously lose Thy-1 expression

(A) We conducted coimmunofluorescence staining for Ecadherin and Thy- 1 in MEF cultures at 2 days after infection with the retroviruses expressing Hnf4a and Foxa3. DNA was stained with DAPI. Scale bar, $100 \mu \mathrm{m}$. (B) The proportion of E-cadherin-positive cells that did not express Thy- 1 at 2 days after the retrovirus infection. The graph shows the average of three independent experiments (mean \pm standard deviation).

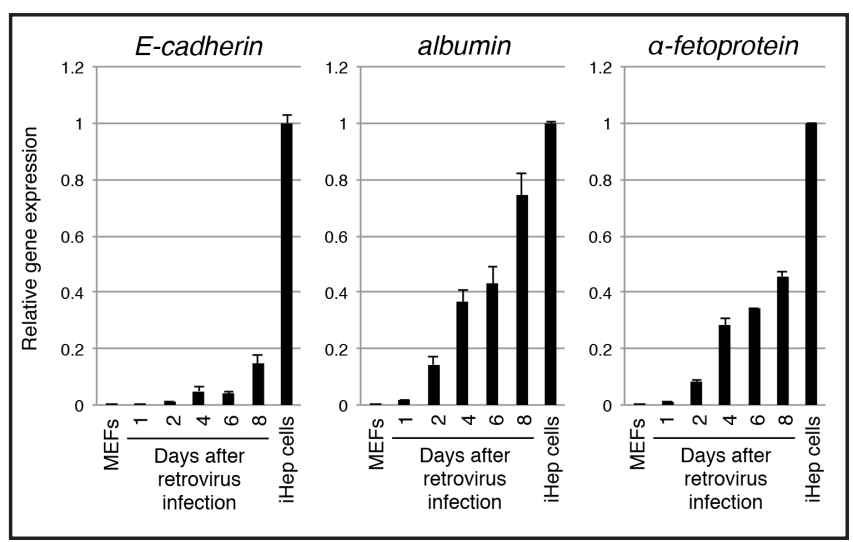

Fig.5 Gene expressions of E-cadherin, albumin, and AFP are detected immediately and then increase gradually in MEF cultures after retrovirus infection

We conducted qPCR analyses on MEF cultures at 1, 2, 4, 6, and 8 days after infection with the retroviruses expressing Hnf4a and Foxa3. MEFs and iHep cells maintained in cultures were analyzed as negative and positive controls, respectively. All data were normalized by the values in iHep cells, and the fold differences are shown. The data represent means \pm standard deviations $(n=3)$.

epithelial cell clusters in MEF cultures could be observed at 36 and $72 \mathrm{~h}$, respectively, after infection with the retroviruses expressing Hnf4a and Foxa3. Similarly, the results of immunofluorescence staining and QPCR analyses revealed that the expression levels of E-cadherin, albumin, and AFP began increasing within 2 days after the retrovirus infection, while the expression of Thy-1 was downregulated in E-cadherin-positive cells. These data indicate that at least 
a small number of MEFs had already lost the properties of fibroblasts at 2 days after the retrovirus infection and newly acquired the properties of iHep cells at almost the same time. Thus, MEFs can initiate conversion into iHep cells more quickly than we expected and are maintained in culture with the ability to proliferate.

Similar to the case for iHep cells, it was reported that direct conversion of MEFs into neuron-like cells and cardiomyocyte-like cells began within a couple of days after the introduction of cell lineage-specific factors ${ }^{10,11)}$. Although dependent on the methods used, conversion of MEFs into iPS cells was also initiated within several days after the introduction of defined transcription factors ${ }^{12)}$. However, obtaining functionally differentiated cells from iPS cell cultures requires a relatively long time for the induction and selection of these cells. Thus, if we could use the direct reprogramming technology, the time required to yield the required cells would be greatly shortened. In actual medical settings, the desired numbers of cells required for particular treatments should be prepared as soon as possible at low cost, and thus, the technology for direct reprogramming will be beneficial as one of the next-generation therapies. We expect that this new technology will be improved to provide safe and increasingly functional cells that can be applicable to the fields of medical science and drug discovery research.

\section{Acknowledgments}

We thank Dr. Toshio Kitamura for sharing reagents, and Kanae Ito, Mayumi Yamamoto, Yuuki Honda, and Chiaki Kaieda for technical assistance.

\section{Sources of funding}

This work was supported in part by Grants-in-Aid for Scientific Research from the Ministry of Education, Culture, Sports, Science and Technology (MEXT) of Japan, a Health and Labour Sciences Research Grant in Japan, The Core Research for Evolutional Science and Technology Program of the Japan Science and Technology Agency, The Life Science Foundation of Japan, The Kurata Memorial Hitachi Science and Technology Foundation, The Inamori Foundation, and The Uehara Memorial Foundation.

\section{Conflict of interests}

None

\section{References}

1)Basma H, Soto-Gutiérrez A, Yannam GR, Liu L, Ito R, Yamamoto T, Ellis E, Carson SD, Sato S, Chen Y, Muirhead D, Navarro-Alvarez N, Wong RJ, RoyChowdhury J, Platt JL, Mercer DF, Miller JD, Strom SC, Kobayashi N, Fox IJ: Differentiation and transplantation of human embryonic stem cell-derived hepatocytes. Gastroenterology. 2009; 136: 990-999.

2) Rashid ST, Corbineau S, Hannan N, Marciniak SJ, Miranda E, Alexander G, Huang-Doran I, Griffin J, Ahrlund-Richter L, Skepper J, Semple R, Weber A, Lomas DA, Vallier L: Modeling inherited metabolic disorders of the liver using human induced pluripotent stem cells. J Clin Invest. 2010; 120: 3127-3136.

3) Si-Tayeb K, Noto FK, Nagaoka M, Li J, Battle MA, Duris C, North PE, Dalton S, Duncan SA: Highly efficient generation of human hepatocyte-like cells from induced pluripotent stem cells. Hepatology. 2010; 51: 297-305.

4)Cayo MA, Cai J, DeLaForest A, Noto FK, Nagaoka M, Clark BS, Collery RF, Si-Tayeb K, Duncan SA: JD induced pluripotent stem cell-derived hepatocytes faithfully recapitulate the pathophysiology of familial hypercholesterolemia. Hepatology. 2012; 56: 2163-2171.

5)Choi SM, Kim Y, Shim JS, Park JT, Wang RH, Leach SD, Liu JO, Deng C, Ye Z, Jang YY: Efficient drug screening and gene correction for treating liver disease using patient-specific stem cells. Hepatology. 2013; 57: 2458-2468.

6) Takebe T, Sekine K, Enomura M, Koike H, Kimura M, Ogaeri T, Zhang RR, Ueno Y, Zheng YW, Koike N, Aoyama S, Adachi Y, Taniguchi H: Vascularized and functional human liver from an iPSC-derived organ bud transplant. Nature. 2013; 499: 481-484.

7)Sekiya S, Suzuki A: Direct conversion of mouse fibroblasts to hepatocyte-like cells by defined factors. Nature. 2011; 475: 390-393.

8)Miura S, Suzuki A: Acquisition of lipid metabolic capability in hepatocyte-like cells directly induced from mouse fibroblasts. Front Cell Dev Biol. 2014; 25: August.

9)Morita S, Kojima T, Kitamura T: Plat-E: an efficient and stable system for transient packaging of retroviruses. Gene Ther. 2000; 7: 1063-1066.

10) leda M, Fu JD, Delgado-Olguin P, Vedantham V, Hayashi Y, Bruneau BG, Srivastava D: Direct reprogramming of fibroblasts into functional cardiomyocytes by defined factors. Cell. 2010; 142: 375-386.

11) Vierbuchen T, Ostermeier A, Pang ZP, Kokubu $Y$, 
Südhof TC, Wernig M: Direct conversion of fibroblasts to functional neurons by defined factors. Nature. 2010; 463: 1035-1041.

12)Polo JM, Anderssen E, Walsh RM, Schwarz BA, Nefzger CM, Lim SM, Borkent M, Apostolou E, Alaei
S, Cloutier J, Bar-Nur O, Cheloufi S, Stadtfeld M, Figueroa ME, Robinton D, Natesan S, Melnick A, Zhu J, Ramaswamy S, Hochedlinger K: A molecular roadmap of reprogramming somatic cells into iPS cells. Cell. 2012; 151: 1617-1632. 